\title{
Marking the occasion
}

\author{
Every issue of NSMB is special to us, but this one marks the end of 2011 with two features that deserve extra attention: \\ a Commentary and an Essay Collection. These pieces and the research articles presented in this issue illustrate what \\ NSMB is all about.
}

$\mathbf{M}$ any of us got into science because we wanted to know how living things work. How do cells and organisms live and multiply? How do they sense and respond to the environment? How do they age and die? How or why do things go wrong, and how can we use the knowledge gained from studying biological systems to fix them?

These are the kinds of broad questions whose answers we feature in NSMB. As stated in the aims and scope of the journal (http://www. nature.com/nsmb/authors/index.html\#aims-scope/), we publish work that provides insight into the molecular mechanisms behind biological processes. Such insight can come from a wide array of approaches, including structural and molecular biology, and also biophysics, biochemistry, cell biology, genetics and, more recently, studies based on large data sets, such as genome-wide analyses. In fact, most of our papers feature a combination of some of these approaches to yield a complete story.

Since its inception, NSMB has always published the best of structural biology. This is partly for historical reasons, because the journal started out as Nature Structural Biology. But it is mainly because structural biology allows us to look at macromolecules at an atomic-level resolution and thereby often informs us about function. The field has progressed quickly, with techniques such as electron microscopy, SAXS and computational methods being integrated into the structural biologist's toolbox. Technical advances in X-ray radiation sources and software improvement for data handling have made crystal structure determination more accessible to a larger number of scientists. An ever-increasing number of structures from EM, NMR and diffraction studies are submitted to the PDB, which just celebrated its 40th anniversary.

In this issue, we mark the occasion with a collection of Essays, entitled "Celebrating Structural Biology." We invited several researchers to share their personal experiences, as members of the structural biology community and of the scientific community at large. And, because 40 is the new 20, we also asked them to tell us about their view of the future of structural biology and the challenges to be overcome. This resulted in a series of diverse, enlightening and thought-provoking pieces, and we hope you will enjoy reading them as much as we did.

In addition to knowledge itself, the most valuable contribution science gives to humankind is the potential to improve the quality of life. A clear case is presented in the Commentary by Moir, Malaspina and Fauci, who take us through recent studies using high-throughput approaches to uncover hundreds of broadly neutralizing antibodies from HIVinfected individuals. Only a handful of naturally occurring antibodies with such properties had previously been described, and the authors explore the implications of these new findings for the development of an effective vaccine against HIV. This is a crucial goal and our best chance to successfully combat the HIV pandemic and the public health threat it represents. Much of the body of work covered in this Commentary builds upon previous structural knowledge of HIV-1 g120 and how it is recognized by these antibodies.

As these two special features illustrate, structural biology remains close to our hearts. In fact, this issue contains eight papers with a major structural component, investigating a wide range of biological questions, including how to reactivate a key enzyme in photosynthesis (nsmb.2171), how to trigger autophagy (nsmb.2165), how to regulate a cellular signaling pathway (nsmb.2152), how to silence chromatin (nsmb.2151) and how to neutralize a deadly virus (nsmb.2150).

But structural data are by no means a prerequisite for publication in NSMB. Work on basic cellular events such as DNA and RNA processes, protein folding and membrane processes has long found a home in our pages, along with studies on small RNAs, chromatin biology and genomic integrity. The mechanistic insight provided by many of these papers often comes from biochemical, molecular and cell biological data. A sample of such papers can be found in this issue, delving into themes such as chromosome fragility (nsmb.2155), DNA damage response (nsmb.2173 and nsmb.2188), telomere biology (nsmb.2172, nsmb.2174 and nsmb.2187), signaling (nsmb.2168), transcriptional regulation (nsmb.2153 and nsmb.2164) and splicing (nsmb.2143).

All of the fields mentioned above are ones we care deeply about, and we look forward to continuing our strong relationship with these communities in 2012, through interactions with our authors and reviewers and also by touching base and forming new connections at conferences and during site visits. In addition, developments in areas such as metabolism, plant biology, microbial pathogenesis and cancer biology now make it possible to address mechanistic questions at the molecular level. Two papers in this issue highlight these burgeoning fields: Lee, Choi et al. (nsmb.2142) investigate how a herpesvirus protein interferes with cellular responses, then use virus-derived peptides to promote p53-dependent cell cycle arrest and xenograft tumor regression. Murga, Campaner et al. (nsmb.2189) demonstrate that the replicative stress induced by oncogenes can be exploited as an Achilles' heel to effectively eliminate lymphomas and pancreatic tumors in mice. These papers are fine examples of applying knowledge derived from the examination of molecular mechanisms to treat cellular processes gone awry.

Finally, we would like to close 2011 by thanking all our reviewers. A list of these invaluable contributors can be found on our website (http://www.nature.com/nsmb/reviewers/index.html). The hard work and high standards of these scientists are crucial for maintaining the quality and rigor that are the hallmark of NSMB papers. We are extremely grateful to work with such a talented group of individuals, who are as committed to science excellence as we are. 